\title{
The Relation of Complete Blood Count Parameters with Metabolic and Clinical Parameters in Overweight and Obese Children
}

\author{
(1) Ayşe Anık1, (1) Elif Çelik1, (1) Ahmet Anık² \\ ${ }^{1}$ Aydın Adnan Menderes University Faculty of Medicine, Department of Pediatrics, Aydın, Turkey \\ ${ }^{2}$ Aydın Adnan Menderes University Faculty of Medicine, Department of Pediatric Endocrinology, Aydın, Turkey
}

\begin{abstract}
Aim: This study aims to assess white blood cell count, platelet count, and platelet indices as a metabolic indicator in overweight, obese and morbidly obese children.

Materials and Methods: One-hundred and thirty overweight, 341 obese, 188 morbidly obese children and 110 controls were enrolled in the study. Anthropometric measurements, pubertal status, complete blood count parameters [white blood cells (WBC), platelet, mean platelet volume (MPV), plateletcrit (PCT), and platelet distribution width (PDW)], WBC differential (neutrophils, lymphocytes, and monocytes), neutrophil/ lymphocyte ratio (NLR) and platelet/lymphocyte ratio (PLR), and serum levels of glucose, lipids, aspartate transaminase (AST), and alanine transaminase (ALT), insulin and thyroid hormones were obtained from the hospital records. Insulin resistance was assessed according to the homeostasis model assessment-insulin resistance (HOMA-IR) index.

Results: WBC, neutrophil, lymphocyte, and monocyte counts were highest in the morbidly obese group followed by the obese, overweight, and healthy groups, respectively. Platelet count, PCT, and PDW were significantly higher in the morbidly obese, obese, and overweight groups compared to the healthy group. However, there was no significant difference between the groups in terms of MPV, NLR, and PLR. WBC, neutrophil, lymphocyte, platelet, PCT, ALT, and triglyceride levels were higher in children with insulin resistance than those without insulin resistance. There was a positive correlation with the neutrophil, lymphocyte, monocyte count, and PCT value, and a negative correlation with the PDW value. Moreover, there was a positive correlation between the HOMA-IR and WBC, neutrophil, lymphocyte count, and PCT.

Conclusion: WBC, neutrophils, lymphocytes, monocytes, platelets, and PCT values increase in childhood obesity, which could point towards low-grade chronic inflammation and this increase in WBC, neutrophils, lymphocytes, and PCT value may be associated with insulin resistance.

Keywords: Children, complete blood count, insulin resistance, obesity
\end{abstract}

\section{Introduction}

Obesity is a medical condition defined as abnormal or excessive fat accumulation in the body to the extent that it may cause a risk to health. Obesity has become a serious health problem across the world, and currently, one in three children in the United States are either overweight or obese. In parallel with the increase in childhood obesity, the frequency of comorbidities such as insulin resistance, type 2 diabetes mellitus, hypertension, non-alcoholic fatty liver, obstructive sleep apnea, and dyslipidemia have 
also increased (1). The close relationship between insulin resistance and obesity indicates that insulin resistance is an important public health issue (2). Cheap and useful markers that can show insulin resistance in the early period are important for the prevention of morbidities related to insulin resistance.

Complete blood count (CBC) is an easy-to-use and cheap test that is frequently used in clinical practice. Neutrophil/ lymphocyte ratio (NLR) and platelet/lymphocyte ratio (PLR) obtained by $C B C$ are simple biomarkers showing inflammation (3-5). Moreover, platelet count, mean platelet volume (MPV), plateletcrit (PCT), and platelet distribution width (PDW) are other biomarkers that indicate systemic inflammation $(6,7)$.

Metabolic syndrome can affect hematologic counts, and hematologic markers can be used for the early detection of individuals at risk of cardiovascular disease $(8,9)$. According to studies conducted on the adult population, white blood cells (WBC), subtypes, and NLR were independently associated with insulin resistance $(10,11)$. In Korean and Chinese adults, obesity, dyslipidemia, glucose intolerance, and hypertension were associated with changes in hematologic parameters $(8,12,13)$. However, few studies have evaluated the relationship between hematological parameters and insulin resistance in childhood, and the results of these studies are inconsistent. Therefore, in this study, we aimed to examine the relationship between CBC parameters and clinical and metabolic parameters in overweight and obese children.

\section{Materials and Methods}

A total of 659 obese or overweight children between the ages of 5 and 18 years who were admitted to the outpatient general paediatrics clinic of our hospital between March 2018 and March 2020 were included in the study. One hundred and ten healthy children with a body mass index (BMI) below the $85^{\text {th }}$ percentile who had a similar age and gender distribution were also enrolled as a control group in this retrospective study.

Those children with type 2 diabetes mellitus, secondary obesity (genetic syndromes, monogenic obesity, endocrine pathology), hypertension, chronic disease or history of acute infection, children with leukopenia $\left(<4 \times 10^{3} / \mathrm{mL}\right)$, leucocytosis $\left(>13.0 \times 10^{3} / \mathrm{mL}\right)$, anaemia or thrombocytopenia $\left(<150 \times 10^{3} / \mathrm{mL}\right)$, a history of drug use that could affect the CBC parameters or bodyweight or incomplete data were excluded from the study.
The age, gender, anthropometric measurements, pubertal status, CBC parameters and biochemical test results of the subjects were obtained from the hospital records. Height was measured using a Harpenden stadiometer with a sensitivity of $0.1 \mathrm{~cm}$, and weight was measured using a SECA scale with a sensitivity of $0.1 \mathrm{~kg}$. BMI was calculated by dividing weight $(\mathrm{kg})$ by height squared $\left(\mathrm{m}^{2}\right)$. Standard deviation scores (SDS) for weight, height, and $\mathrm{BMI}$ were calculated with the online calculator for paediatric endocrinologists (child metrics) using a reference created for the Turkish population by Neyzi et al. $(14,15)$. Findings for pubertal development were evaluated according to Tanner and Whitehouse staging (16). A testicular volume of $\geq 4 \mathrm{~mL}$ in males and breast development $\geq$ stage 2 in females were considered to be findings of puberty.

Subjects with a BMI over the $85^{\text {th }}$ but less than the $95^{\text {th }}$ percentile for age and gender are defined as overweight, and those with a BMI higher than the $95^{\text {th }}$ percentile are defined as obese. Subjects with a BMI higher than the $99^{\text {th }}$ percentile are defined as severely obese (17). Blood samples were taken after 10-12 hours of night fasting. Serum levels of glucose, lipids, aspartate transaminase (AST), and alanine transaminase (ALT) were measured by a spectrophotometric method; insulin and thyroid hormones were measured by chemiluminescent microparticle immunoassay (Abbot-Architect i2000SR). CBC parameters (WBC, platelet, PDW, MPV, PCT) and WBC differential (neutrophils, lymphocytes, monocytes) were measured by flow impedance, laser light scattering, and flow cytometry methods (Mindray, BC6800). NLR and PLR were obtained by dividing the number of neutrophils and platelets by lymphocytes, respectively. Insulin resistance was assessed according to the homeostasis model assessment-insulin resistance (HOMA-IR) index. Different cut-off values for prepubertal and pubertal stages were used to determine the status of insulin resistance (prepubertal $>2.5$, pubertal >4) (18).

The study was initiated upon approval form the local ethics committee of Aydın Adnan Menderes University Faculty of Medicine, in light of the Helsinki Declaration (2020/98).

\section{Statistical Analysis}

Statistical analysis of the data were conducted with SPSS 21.0 (SPSS Inc., Chicago, IL, USA). The distribution of data was evaluated with the Kolmogorov-Smirnov test and histograms. Clinical data were presented as number (\%), mean \pm standard deviation for normal distribution, and median (25p-75p-value) for data that were not distributed 
normally. Categorical variables were compared using the chi-square test. Between study groups, the data obtained were compared by using Student's t-test (for normally distributed data), and Mann-Whitney $U$ test (for nonnormally distributed data). The correlations between the independent parameters were investigated with Pearson's correlation analysis or Spearman's rank correlation and partial correlation analysis with respect to the effect of age, gender, and pubertal status. A p-value of less than 0.05 was considered statistically significant.

\section{Results}

A total of 110 healthy (45 males; median age 13.5 years), 130 overweight ( 58 males; median age 12.5 years), 341 obese (161 males; median age 12.5 years), and 188 morbidly obese (66 males; median age 14.0 years) subjects were included in this study. There were no significant differences between the groups in terms of age, gender, or pubertal status (Table I).

WBC, neutrophil, lymphocyte, and monocyte counts were highest in the morbidly obese group, followed by the obese, overweight, and healthy group, respectively $(p<0.001)$. Platelet count, PCT, and PDW were significantly higher in the morbidly obese, obese, and overweight groups compared to the healthy group. However, there was no significant difference between the groups in terms of MPV, NLR, and PLR (Table II).

There was a significant difference in fasting serum insulin and HOMA-IR levels of the morbidly obese, obese, and overweight patients. Serum HDL levels were significantly lower in the morbidly obese children than in those of the other groups (Table III).

When obese children were compared according to insulin resistance status, WBC $(p<0.001)$, neutrophil $(p=0.002)$, lymphocyte $(p=0.001)$, platelet $(p=0.034), P C T(p=0.05)$, ALT $(p<0.01)$, and triglyceride $(p<0.001)$ levels were higher in those children with insulin resistance than in those without insulin resistance (Table IV).

There was a positive correlation between the BMI-SDS and WBC, neutrophil, lymphocyte, monocyte, platelet count, $N L R$, and PCT value $(p<0.001)$, and a negative correlation with the PDW value $(p=0.001)$. In partial correlation analysis, similar correlations were found between the BMI-SDS and blood count parameters except for platelets and NLR (Table $\mathrm{V})$. Moreover, there was a positive correlation between the HOMA-IR and WBC $(p=0.014)$, neutrophil $(p=0.003)$, and NLR ( $p=0.02)$. Additionally, in partial correlation analysis, there was a positive correlation between the HOMA-IR and lymphocyte $(p<0.001)$ and PCT $(p=0.006)$ (Table VI).

Table I. The clinical characteristics of the study population

\begin{tabular}{|c|c|c|c|c|c|}
\hline & $\begin{array}{l}\text { Healthy } \\
\text { (Group 1) } \\
(n=110)\end{array}$ & $\begin{array}{l}\text { Overweight } \\
\text { (Group 2) } \\
(\mathrm{n}=130)\end{array}$ & $\begin{array}{l}\text { Obese } \\
\text { (Group 3) } \\
(n=341)\end{array}$ & $\begin{array}{l}\text { Morbid obese } \\
\text { (Group 4) } \\
(\mathrm{n}=188)\end{array}$ & p-value* \\
\hline \multirow{2}{*}{ Age (year) } & 13.5 & 12.5 & 12.5 & 14.0 & \multirow{2}{*}{0.056} \\
\hline & $(10.2-15.3)$ & $(10.6-14.0)$ & $(10.0-14.5)$ & $(10.0-15.7)$ & \\
\hline \multicolumn{6}{|l|}{ Gender } \\
\hline Female (\%) & $65(59)$ & $72(55)$ & $180(53)$ & $122(65)$ & \multirow{2}{*}{0.055} \\
\hline Male (\%) & $45(41)$ & $58(45)$ & $161(47)$ & $66(35)$ & \\
\hline \multicolumn{6}{|l|}{ Puberty } \\
\hline Prepubertal (\%) & $27(25)$ & $36(28)$ & $100(29)$ & $46(24)$ & \multirow{2}{*}{0.595} \\
\hline Pubertal (\%) & $83(75)$ & $94(72)$ & $241(71)$ & $142(76)$ & \\
\hline Weight-SDS & $\begin{array}{l}-0.20 \\
(-0.74-0.36)\end{array}$ & $\begin{array}{l}1.80 \\
(1.30-2.13)\end{array}$ & $\begin{array}{l}2.64 \\
(2.18-3.07)\end{array}$ & $\begin{array}{l}3.84 \\
(3.43-4.37)\end{array}$ & $<0.01^{a}$ \\
\hline Height-SDS & $\begin{array}{l}0.09 \\
(-0.89-0.75)\end{array}$ & \begin{tabular}{|l}
0.74 \\
$(-0.29-1.48)$ \\
\end{tabular} & $\begin{array}{l}0.65 \\
(-0.09-1.37) \\
\end{array}$ & $\begin{array}{l}0.63 \\
(-0.13-1.49)\end{array}$ & $<0.01^{\text {b }}$ \\
\hline BMI-SDS & $\begin{array}{l}-0.33 \\
(-0.75-0.20)\end{array}$ & $\begin{array}{l}1.76 \\
(1.50-1.89)\end{array}$ & $\begin{array}{l}2.53 \\
(2.27-2.80)\end{array}$ & $\begin{array}{l}3.34 \\
(3.14-3.68)\end{array}$ & $<0.01^{a}$ \\
\hline \multicolumn{6}{|c|}{$\begin{array}{l}\text { Continuous variables are expressed as median (25-75p), while the categorical variables are expressed as number (percentage), *Kruskal-Wallis test } \\
\text { aGroup } 1 \text { \& Group 2, Group } 1 \text { \& Group 3, Group } 1 \text { \& Group 4, Group } 2 \text { \& Group3, Group } 2 \text { \& Group 4, Group } 3 \text { \& Group 4, p<0.001. } \\
\text { bGroup } 1 \text { \& Group2, Group } 1 \text { \& Group 3, Group } 1 \text { \& Group 4, p<0.001. } \\
\text { BMI: Body mass index, SDS: Standard deviation score }\end{array}$} \\
\hline
\end{tabular}




\section{Discussion}

In this study, WBC, neutrophils, lymphocytes, monocytes, platelet count, MPV, and PCT values were significantly higher in the obese children. Also, there was a positive correlation between BMI, HOMA-IR and WBC, neutrophils, lymphocytes, and PCT.

\section{White Blood Cells}

Studies point towards low-grade chronic inflammation in adipose tissue as the initiating mechanism of obesity-related complications (19). Macrophage infiltration in adipose tissues plays a leading role in the development of chronic inflammation (20). It was shown that pro-inflammatory cytokines secreted from activated macrophages, such as tumour necrosis factor-alpha and interleukin 6, disrupt the autocrine and paracrine effect of insulin in adipocytes $(21,22)$. As well as macrophages, the effects of other leucocyte subgroups in adipose tissue inflammation were shown (23). Experimental studies showed an increase in neutrophil count in adipose tissues even in the first few days of a highfat diet, and this finding led to the idea that inflammation is seen in the early stages of obesity (24). On the other hand, studies investigating the relationship between childhood obesity and leucocyte subgroups and insulin resistance are limited. In this study, we found that WBC, neutrophil, and lymphocyte count were higher in obese children with insulin resistance compared to obese children without insulin resistance. Furthermore, a positive correlation was shown between HOMA-IR and WBC, neutrophil, and lymphocyte count. These findings seem to support the idea of the effect

\begin{tabular}{|c|c|c|c|c|c|}
\hline & $\begin{array}{l}\text { Healthy } \\
\text { (Group 1) } \\
(n=110)\end{array}$ & $\begin{array}{l}\text { Overweight } \\
\text { (Group 2) } \\
\text { (n=130) }\end{array}$ & $\begin{array}{l}\text { Obese } \\
\text { (Group 3) } \\
(n=341)\end{array}$ & $\begin{array}{l}\text { Morbid obese } \\
\text { (Group 4) } \\
(n=188)\end{array}$ & p-value* \\
\hline WBC $\left(10^{3} / \mu \mathrm{L}\right)$ & $\begin{array}{l}7,015 \\
(5,867-8,140)\end{array}$ & $\begin{array}{l}8,270 \\
(7,223-9,725)\end{array}$ & $\begin{array}{l}8,570 \\
(7,375-9,810)\end{array}$ & $\begin{array}{l}9,135 \\
(7,805-10,288)\end{array}$ & $<0.001^{\mathrm{a}}$ \\
\hline Neutrophil $\left(10^{3} / \mu \mathrm{L}\right)$ & $\begin{array}{l}3,745 \\
(2,988-4,763)\end{array}$ & $\begin{array}{l}4,295 \\
(3,743-5,295)\end{array}$ & $\begin{array}{l}4,530 \\
(3,660-5,715)\end{array}$ & $\begin{array}{l}5,135 \\
(4,180-6,123)\end{array}$ & $<0.001^{b}$ \\
\hline Lymphocyte $\left(10^{3} / \mu \mathrm{L}\right)$ & $\begin{array}{l}2,375 \\
(2,068-, 2878)\end{array}$ & $\begin{array}{l}2,960 \\
(2,503-3,600)\end{array}$ & $\begin{array}{l}3,060 \\
(2,515-3,570)\end{array}$ & $\begin{array}{l}3,085 \\
(2,543-3,465)\end{array}$ & $<0.001^{c}$ \\
\hline Monocyte $\left(10^{3} / \mu \mathrm{L}\right)$ & $\begin{array}{l}440 \\
(360-560)\end{array}$ & $\begin{array}{l}520 \\
(430-650)\end{array}$ & $\begin{array}{l}520 \\
(440-600)\end{array}$ & $\begin{array}{l}535 \\
(440-658)\end{array}$ & $<0.001^{d}$ \\
\hline$N / L$ & $\begin{array}{l}1.57 \\
(1.16-2.07)\end{array}$ & $\begin{array}{l}1.44 \\
(1.18-1.81)\end{array}$ & $\begin{array}{l}1.48 \\
(1.18-1.96)\end{array}$ & $\begin{array}{l}1.76 \\
(1.32-2.11)\end{array}$ & 0.370 \\
\hline Platelet $\left(10^{3} / \mu \mathrm{L}\right)$ & $\begin{array}{l}286.0 \\
(249.5-336.5)\end{array}$ & $\begin{array}{l}322.5 \\
(289.5-365.5)\end{array}$ & $\begin{array}{l}332.0 \\
(287.0-383.0)\end{array}$ & $\begin{array}{l}331.5 \\
(289.0-388.8)\end{array}$ & $<0.001^{e}$ \\
\hline MPV & $\begin{array}{l}9.3 \\
(8.7-9.8)\end{array}$ & $\begin{array}{l}9.7 \\
(8.9-10.2)\end{array}$ & $\begin{array}{l}9.3 \\
(8.7-9.9)\end{array}$ & $\begin{array}{l}9.4 \\
(8.8-10.0)\end{array}$ & $0.045^{f}$ \\
\hline PDW & $\begin{array}{l}15.9 \\
(15.6-16.1)\end{array}$ & $\begin{array}{l}15.8 \\
(15.5-16.1)\end{array}$ & $\begin{array}{l}15.7 \\
(15.4-15.9)\end{array}$ & $\begin{array}{l}15.7 \\
(15.5-16.0)\end{array}$ & $0.001^{\mathrm{g}}$ \\
\hline РCT & $\begin{array}{l}0.26 \\
(0.23-0.31)\end{array}$ & $\begin{array}{l}0.31 \\
(0.27-0.35)\end{array}$ & $\begin{array}{l}0.31 \\
(0.27-0.35)\end{array}$ & $\begin{array}{l}0.30 \\
(0.27-0.36)\end{array}$ & $<0.001^{h}$ \\
\hline P/L & $\begin{array}{l}0.12 \\
(0.09-0.14)\end{array}$ & $\begin{array}{l}0.10 \\
(0.08-0.12)\end{array}$ & $\begin{array}{l}0.11 \\
(0.09-0.13)\end{array}$ & $\begin{array}{l}0.11 \\
(0.10-0.13)\end{array}$ & 0.236 \\
\hline \multicolumn{6}{|c|}{ 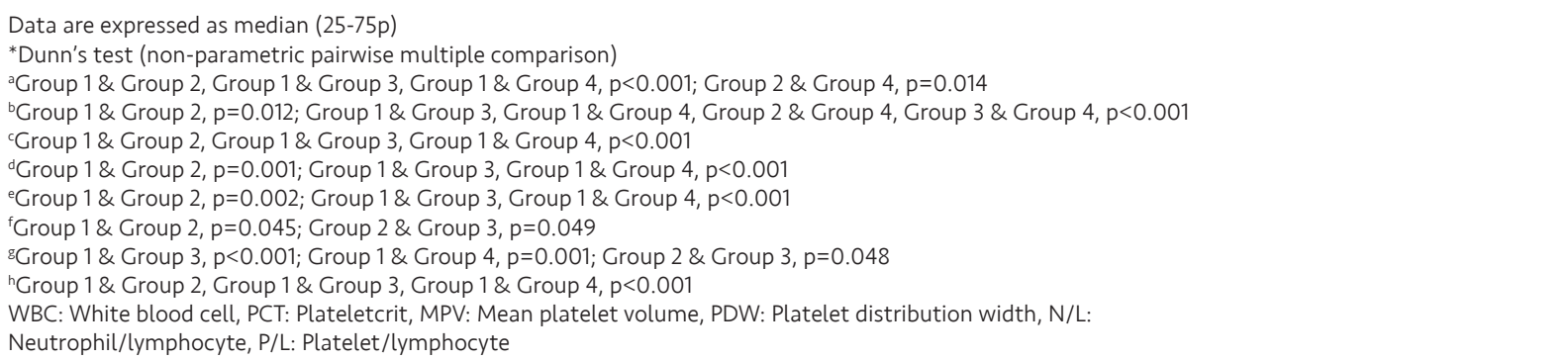 } \\
\hline
\end{tabular}


Table III. The laboratory characteristics of the study population

\begin{tabular}{|c|c|c|c|c|}
\hline & $\begin{array}{l}\text { Overweight } \\
\text { (Group 2) } \\
(\mathrm{n}=130)\end{array}$ & $\begin{array}{l}\text { Obese } \\
\text { (Group 3) } \\
(n=341)\end{array}$ & $\begin{array}{l}\text { Morbid obese } \\
\text { (Group 4) } \\
(n=188)\end{array}$ & p-value* \\
\hline Glucose (mg/dL) & $\begin{array}{l}88 \\
(81-92)\end{array}$ & $\begin{array}{l}87 \\
(81-92)\end{array}$ & $\begin{array}{l}86 \\
(82-91)\end{array}$ & 0.776 \\
\hline Insulin ( $\mu \mid \mathrm{I} / \mathrm{mL})$ & \begin{tabular}{|l}
11.6 \\
$(8.7-15.8)$ \\
\end{tabular} & \begin{tabular}{|l}
13.8 \\
$(9.9-19.5)$
\end{tabular} & $\begin{array}{l}17.0 \\
(10.8-24.9)\end{array}$ & $<0.001^{a}$ \\
\hline HOMA-IR & $\begin{array}{l}2.43 \\
(1.84-3.40)\end{array}$ & $\begin{array}{l}2.95 \\
(2.09-4.30)\end{array}$ & $\begin{array}{l}3.64 \\
(2.24-5.33)\end{array}$ & $<0.001^{b}$ \\
\hline AST (U/L) & $\begin{array}{l}21 \\
(18-26)\end{array}$ & $\begin{array}{l}22 \\
(18-22)\end{array}$ & $\begin{array}{l}21 \\
(17-26)\end{array}$ & 0.316 \\
\hline ALT (U/L) & $\begin{array}{l}18 \\
(14-26)\end{array}$ & \begin{tabular}{|l}
21 \\
$(16-30)$
\end{tabular} & $\begin{array}{l}21 \\
(16-33)\end{array}$ & $0.011^{c}$ \\
\hline $\mathrm{TC}(\mathrm{mg} / \mathrm{dL})$ & \begin{tabular}{|l}
164 \\
$(149-186)$ \\
\end{tabular} & \begin{tabular}{|l}
163 \\
$(145-182)$ \\
\end{tabular} & $\begin{array}{l}162 \\
(144-179)\end{array}$ & 0.489 \\
\hline $\mathrm{HDL}(\mathrm{mg} / \mathrm{dL})$ & $\begin{array}{l}50 \\
(45-59)\end{array}$ & $\begin{array}{l}47 \\
(40-56)\end{array}$ & $\begin{array}{l}45 \\
(39-52)\end{array}$ & $0.01^{d}$ \\
\hline TG (mg/dL) & $\begin{array}{l}93 \\
(66-123) \\
\end{array}$ & $\begin{array}{l}94 \\
(70-130) \\
\end{array}$ & $\begin{array}{l}95 \\
(67-137)\end{array}$ & 0.384 \\
\hline LDL (mg/dL) & \begin{tabular}{|l}
93 \\
$(80-112)$
\end{tabular} & $\begin{array}{l}92 \\
(75-107)\end{array}$ & $\begin{array}{l}92 \\
(76-113)\end{array}$ & 0.668 \\
\hline fT4 (ng/dL) & $\begin{array}{l}0.97 \\
(0.91-1.05)\end{array}$ & \begin{tabular}{|l}
1.0 \\
$(0.92-1.09)$ \\
\end{tabular} & $\begin{array}{l}0.98 \\
(0.89-1.07)\end{array}$ & 0.075 \\
\hline TSH (mIU/L) & $\begin{array}{l}1.92 \\
(1.24-2.75)\end{array}$ & $\begin{array}{l}2.10 \\
(1.47-2.79)\end{array}$ & $\begin{array}{l}2.27 \\
(1.60-3.03)\end{array}$ & 0.079 \\
\hline \multicolumn{5}{|c|}{ 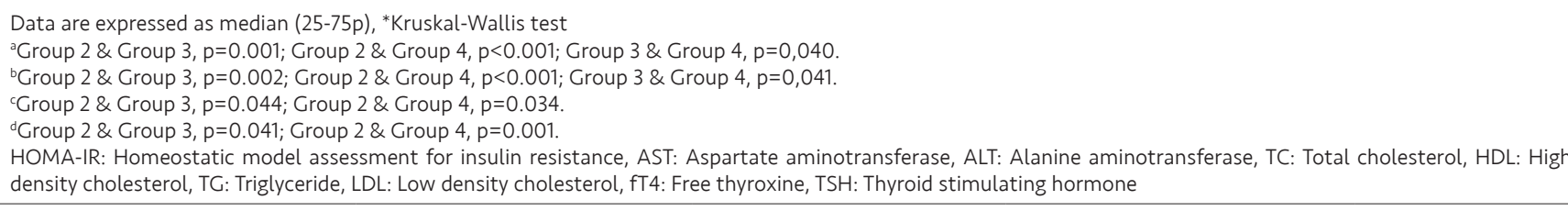 } \\
\hline
\end{tabular}

of adipose tissue inflammation on insulin resistance and white blood cells.

In a study of 656 adults at high risk of developing type 2 diabetes mellitus, a positive correlation was found between leucocyte subgroups (neutrophils, lymphocytes, monocytes), NLR, and HOMA-IR (10). In contrast to that study, we did not see a correlation between monocyte count and NLR and HOMA-IR in our study; this difference was thought to be related to the difference in population and age group.

We could not find a significant relationship between $B M I, H O M A-I R$, and NLR, although it is well known that an increase in NLR and PLR in chronic inflammation is a marker of immune response $(25,26)$. The lack of a significant relation between NLR and BMI in our study could be related to an increase in lymphocyte count together with a neutrophil count. Similar to our study, Marginean et al. (3) and Bahadir et al. (27) did not find a relation between NLR and obesity in children and adults. Nevertheless, it was shown that NLR is a marker suggesting inflammation in studies of obese patients with comorbidity. A positive correlation was found between NLR and carotid intima-media thickness in adult patients who were candidates for bariatric surgery (28). In another study, it was reported that NLR was higher in obese children who have obstructive sleep apnoea compared to those who do not (4). Vuong et al. (29) assessed the relation between blood count and waist circumference in 6,766 adults and showed that there is a positive correlation between neutrophil, lymphocyte, and monocyte count with waist circumference. Furthermore, in the same study, they saw that the percentage of neutrophils increased; however, lymphocyte and monocyte percentage decreased with increasing waist circumference (29). Similarly, in our study, the neutrophil percentage increased with increasing BMI; 
Table IV. The clinical and laboratory characteristics of the overweight and obese patients with and without insulin resistance

\begin{tabular}{|c|c|c|c|}
\hline & \multicolumn{2}{|c|}{ Insulin Resistance } & \multirow[b]{2}{*}{ p-value* } \\
\hline & $\begin{array}{l}\text { No } \\
(n=424)\end{array}$ & $\begin{array}{l}\text { Yes } \\
(n=227)\end{array}$ & \\
\hline Age (year) & \begin{tabular}{|l}
12.1 \\
$(9.0-14.6)$
\end{tabular} & \begin{tabular}{|l}
3.0 \\
$(11.0-15.0)$
\end{tabular} & 0.002 \\
\hline $\begin{array}{l}\text { Gender (\%) } \\
\text { Male } \\
\text { Female }\end{array}$ & $\begin{array}{l}199(47) \\
225(53) \\
\end{array}$ & $\begin{array}{l}96(42) \\
131(58) \\
\end{array}$ & 0.257 \\
\hline $\begin{array}{l}\text { Puberty (\%) } \\
\text { Prepubertal } \\
\text { Pubertal }\end{array}$ & $\begin{array}{l}129(30) \\
295(70)\end{array}$ & $\begin{array}{l}50(22) \\
177(78) \\
\end{array}$ & 0.022 \\
\hline BMI-SDS & \begin{tabular}{|l|}
2.53 \\
$(2.01-3.0)$ \\
\end{tabular} & $\begin{array}{l}2.83 \\
(2.38-3.32) \\
\end{array}$ & $<0.001$ \\
\hline WBC $\left(10^{3} / \mu \mathrm{L}\right)$ & \begin{tabular}{|l|}
8,550 \\
$(7,200-9,770)$
\end{tabular} & $\begin{array}{l}9,070 \\
(7,980-10,420) \\
\end{array}$ & $<0.001$ \\
\hline Neutrophil $\left(10^{3} / \mu \mathrm{L}\right)$ & $\begin{array}{l}4,540 \\
(3,680-5,550)\end{array}$ & $\begin{array}{l}4,795 \\
(3,990-5,970)\end{array}$ & 0.002 \\
\hline Lymphocyte $\left(10^{3} / \mu \mathrm{L}\right)$ & $\begin{array}{l}2,970 \\
(2,460-3,490)\end{array}$ & \begin{tabular}{|l|}
3,140 \\
$(2,680-2,670)$ \\
\end{tabular} & 0.001 \\
\hline Monocyte $\left(10^{3} / \mu \mathrm{L}\right)$ & \begin{tabular}{|l}
520 \\
$(430-610)$ \\
\end{tabular} & $\begin{array}{l}520 \\
(450-640) \\
\end{array}$ & 0.244 \\
\hline Platelet $\left(10^{3} / \mu \mathrm{L}\right)$ & $\begin{array}{l}327 \\
(283-382) \\
\end{array}$ & \begin{tabular}{|l|}
341 \\
$(297-441)$
\end{tabular} & 0.034 \\
\hline РСТ (\%) & $\begin{array}{l}0.31 \\
(0.27-0.35)\end{array}$ & \begin{tabular}{|l}
0.32 \\
$(0.28-0.35)$ \\
\end{tabular} & 0.055 \\
\hline MPV (fL) & \begin{tabular}{|l}
9.4 \\
$(8.8-10.1)$ \\
\end{tabular} & \begin{tabular}{|l}
9.3 \\
$(8.7-10.0)$ \\
\end{tabular} & 0.250 \\
\hline PDW & $\begin{array}{l}15.7 \\
(15.5-16.0)\end{array}$ & \begin{tabular}{|l}
5.7 \\
$(15.4-15.9)$ \\
\end{tabular} & 0.037 \\
\hline N/L & $\begin{array}{l}1.53 \\
(1.20-2.01) \\
\end{array}$ & $\begin{array}{l}1.59 \\
(1.22-1.99) \\
\end{array}$ & 0.706 \\
\hline P/L & \begin{tabular}{|l}
0.11 \\
$(0.09-0.13)$
\end{tabular} & $\begin{array}{l}0.11 \\
(0.09-0.13) \\
\end{array}$ & 0.353 \\
\hline Glucose (mg/dL) & \begin{tabular}{|l|}
85 \\
$(80-90)$ \\
\end{tabular} & \begin{tabular}{|l}
91 \\
$(85-95)$ \\
\end{tabular} & $<0.001$ \\
\hline Insulin $(\mu \mid \mathrm{U} / \mathrm{mL})$ & \begin{tabular}{|l|}
11.0 \\
$(8.2-14.4)$ \\
\end{tabular} & $\begin{array}{l}23.2 \\
(18.9-28.6) \\
\end{array}$ & $<0.001$ \\
\hline HOMA-IR & \begin{tabular}{|l}
2.3 \\
$(1.7-3.1)$ \\
\end{tabular} & \begin{tabular}{|l}
5.1 \\
$(4.3-6.7)$ \\
\end{tabular} & $<0.001$ \\
\hline AST (U/L) & $\begin{array}{l}22 \\
(17-16) \\
\end{array}$ & $\begin{array}{l}22 \\
(17-28) \\
\end{array}$ & 0.809 \\
\hline ALT (U/L) & \begin{tabular}{|l}
19 \\
$(15-27)$ \\
\end{tabular} & \begin{tabular}{|l}
24 \\
$(17-35)$ \\
\end{tabular} & $<0.01$ \\
\hline TC (mg/dL) & \begin{tabular}{|l}
161 \\
$(145-181)$
\end{tabular} & \begin{tabular}{|l|l|}
165 \\
$(147-182)$
\end{tabular} & 0.246 \\
\hline $\mathrm{HDL}(\mathrm{mg} / \mathrm{dL})$ & \begin{tabular}{|l}
49 \\
$(42-57)$ \\
\end{tabular} & $\begin{array}{l}44 \\
(38-52) \\
\end{array}$ & $<0.001$ \\
\hline TG (mg/dL) & \begin{tabular}{|l}
89 \\
$(66-120)$
\end{tabular} & \begin{tabular}{|l}
105 \\
$(79-146)$ \\
\end{tabular} & $<0.001$ \\
\hline LDL (mg/dL) & \begin{tabular}{|l}
91 \\
$(77-109)$
\end{tabular} & $\begin{array}{l}94 \\
(77-110)\end{array}$ & 0.214 \\
\hline
\end{tabular}

Continuous variables are expressed as median (25-75p), while the categorical variables are expressed as number (percentage)

* Mann-Whitney U test

BMI: Body mass index, WBC: White blood cell, PCT: Plateletcrit, MPV: Mean platelet volume, PDW: Platelet distribution width, N/L: Neutrophil/lymphocyte, P/L: Platelet/lymphocyte, HOMA-IR: Homeostatic model assessment for insulin resistance, AST: Aspartate aminotransferase, ALT: Alanine aminotransferase, TC: Total cholesterol, HDL: High density cholesterol, TG: Triglyceride, LDL: Low density cholestero lymphocyte and monocyte count also increased in numbers, but their percentages in white blood cell distribution decreased, which means they did not increase as much as neutrophils. We saw that the percentage of neutrophils increased, and the percentage of lymphocytes decreased with increasing BMI, and NLR also increased; however, this increase did not reach statistical significance.

\section{Platelets}

Similar to an increase in WBC, platelet count also increases in acute and chronic inflammation due to secreted cytokines (30). Moreover, it is thought that thrombopoietin, one of the adipokines secreted from omental adipose tissue, may be responsible for both the increase in platelet count and insulin resistance (31). In addition, insulin resistance shortens platelet life, and that causes an increase in the number of platelets (32).

Similar to the results of our study, Arslan and Makay (33) reported that MPV was significantly higher in 128 obese children compared to normal-weight children. The different result from our study was that they reported a positive correlation between MPV and HOMA-IR (33). In 330 prepubertal children (125 normal weight, 205 overweight), platelet count was significantly higher in overweight children, and MPV was significantly lower in overweight children with metabolic syndrome (34). There was a negative correlation between MPV and HOMA-IR (34). There are also studies reporting higher platelet count, and lower MPV in adult obese subjects $(35,36)$. In another study, in which children were assessed in three groups as normal weight, obese with insulin resistance and obese without insulin resistance, no difference was found between the groups in terms of platelet count, MPV and PDW values (37). Similar to our results, in an adult study, PDW decreased with increasing $\mathrm{BMI}$, and it was shown that the risk of having lower PDW increased with an increase in BMI (38). In contrast, some studies report higher PDW in adults with metabolic syndrome (6). Also, some studies reported no significant difference in the platelet indices of obese and normal-weight children (37). Different results were reported in studies assessing the relation between obesity, metabolic syndrome, and platelet indices such as MPV and PDW (39-42). To the best of our knowledge, this is the first study assessing the relation between PCT and childhood obesity. PCT provides more accurate information about platelet mass (1). PCT is the volume occupied by platelets in the blood as a percentage and it is calculated according to the formula PCT=platelet count $\times$ MPV/10,000 (43). We 
Table V. Correlation analysis of body mass index SDS with complete blood count parameters

\begin{tabular}{|l|l|l|l|l|}
\hline & Spearman's rho & $\mathbf{p}^{*}$ & Partial correlation & $\mathbf{p}^{* *}$ \\
\hline WBC & 0.260 & $<0.001$ & 0.230 & $<0.001$ \\
\hline Neutrophil & 0.250 & $<0.001$ & 0.196 & $<0.001$ \\
\hline Lymphocyte & 0.165 & $<0.001$ & 0.135 & $<0.001$ \\
\hline Monocyte & 0.146 & $<0.001$ & 0.147 & $<0.001$ \\
\hline Platelet & 0.125 & $<0.001$ & 0.023 & 0.530 \\
\hline PCT & 0.159 & $<0.001$ & 0.206 & $<0.001$ \\
\hline MPV & 0.009 & 0.807 & 0.059 & 0.100 \\
\hline PDW & -0.137 & $<0.001$ & -0.125 & 0.001 \\
\hline N/L & 0.097 & 0.007 & 0.048 & 0.186 \\
\hline P/L & -0.044 & 0.222 & 0.024 & 0.508 \\
\hline SPPa & & \\
\hline
\end{tabular}

*Spearman's correlation analysis, ${ }^{* *}$ Partial correlation analysis (age, gender and pubertal status)

WBC: White blood cell, PCT: Plateletcrit, MPV: Mean platelet volume, PDW: Platelet distribution width, N/L: Neutrophil/lymphocyte, P/L: Platelet/lymphocyte

Table VI. Correlation analysis of HOMA-IR with complete blood count parameters

\begin{tabular}{|l|l|l|l|l|}
\hline & Spearman's rho & $\mathbf{p}^{*}$ & Partial correlation & $\mathbf{p}^{* *}$ \\
\hline WBC & 0.107 & 0.014 & 0.150 & 0.001 \\
\hline Neutrophil & 0.130 & 0.003 & 0.089 & 0.042 \\
\hline Lymphocyte & 0.024 & 0.582 & 0.164 & $<0.001$ \\
\hline Monocyte & 0.047 & 0.281 & 0.062 & 0.160 \\
\hline Platelet & -0.009 & 0.846 & -0.056 & 0.206 \\
\hline PCT & 0.045 & 0.303 & 0.121 & 0.006 \\
\hline MPV & 0.066 & 0.133 & -0.070 & 0.109 \\
\hline PDW & 0.039 & 0.377 & -0.061 & 0.163 \\
\hline N/L & 0.102 & 0.020 & -0.061 & 0.166 \\
\hline P/L & -0.019 & -0.057 & 0.198 \\
\hline $\begin{array}{l}\text { *Spearman's correlation analysis, **Partial correlation analysis (age, gender and pubertal status) } \\
\text { HOMA-IR: Homeostatic model assessment for insulin resistance, WBC: White blood cell, PCT: Plateletcrit, MPV: Mean platelet volume, PDW: Platelet distribution width, } \\
\text { N/L: Neutrophil/lymphocyte, P/L: Platelet/lymphocyte }\end{array}$ & \\
\hline
\end{tabular}

observed a positive correlation between PCT and BMI and HOMA-IR in our study. In a study of young adult morbidly obese patients, it was found that PCT was higher in obese patients compared to normal-weight patients, similar to our study, and no significant difference was found in MPV and PDW values (35). Han et al. (44) reported that platelet count and PCT were positively associated with BMI and body fat mass, but there were no significant associations between the other platelet indices (MPV, PDW), and body fat. There are also adult studies reporting similar results between PCT and BMI $(45,46)$.

Although PLR, as well as NLR, are known as chronic inflammation-related markers, no relation was found between PLR with being overweight/obesity and insulin resistance in our study (47). PLR was found to be significantly higher in morbidly obese adult patients compared to normal-weight patients (35). Erdim et al. (4) found that PLR was significantly higher in 127 obese children with a hypopnea index of $>5$ compared to other children. Additionally, in another study, an increase in platelet and lymphocyte count was seen in obese children; however, no increase was seen in PLR, similar to our study (3). A study of 600 adult obese patients, assessing the relation between serum inflammatory markers and visceral adiposity, showed that there is a stronger correlation between WBC and highsensitive C-reactive protein and visceral adipose tissue compared to PLR and NLR (48). These inconsistent results 
were thought to be related to the different ages of the subjects included in the studies and the accompanying complications of obesity.

Obesity is an independent risk factor for having higher cell counts in children, specifically WBC, neutrophils, and platelets (49). In the present study, when subjects were compared according to their pubertal status, WBC, neutrophil, lymphocyte, and monocyte counts were higher in the obese groups than those of the healthy subjects in both prepubertal and pubertal children (data is not shown). However, there was no difference between the groups in terms of PLT, MPV, RDW, N/L and $P / L$ in prepubertal children, and MPV and P/L in pubertal children. The lack of significance for these parameters is thought to be due to the relatively small sample size of the subgroups.

\section{Study Limitations}

Firstly, caution should be taken about causative comments regarding the observed findings due to the crosssectional design. Secondly, infections cannot be strictly ruled out, although those patients with a suspicion of infection and WBC $>13.5 \times 10^{3} / \mathrm{mL}$ were excluded from the study. Furthermore, despite the statistically significant p-values in correlation analysis, the correlation coefficients were low. We thought that the main reason for this situation was related to the large sample size. The strengths of the study were that the sampling size was large, the cases were compared in four different groups as normal, overweight, obese, and morbidly obese, and also, the study was performed in a single centre that has a standard protocol.

\section{Conclusion}

In this study, it was shown that WBC, neutrophils, lymphocytes, monocytes, platelets, and PCT values increase in childhood obesity, which could point towards low-grade chronic inflammation and also an increase in WBC, neutrophils, lymphocytes and PCT values might be associated with insulin resistance.

\section{Ethics}

Ethics Committee Approval: The study was initiated upon approval form the local ethics committee of Aydın Adnan Menderes University Faculty of Medicine, in light of the Helsinki Declaration (2020/98).

Informed Consent: Retrospective study.

Peer-review: Externally peer-reviewed.

\section{Authorship Contributions}

Concept: A.A., Data Collection or Processing: Ay.A., E.Ç., Analysis or Interpretation: A.A., Writing: Ay.A., E.Ç.

Conflict of Interest: No conflict of interest was declared by the authors.

Financial Disclosure: The authors declared that this study received no financial support.

\section{References}

1. Kumar S, Kelly AS. review of childhood obesity: from epidemiology, etiology, and comorbidities to clinical assessment and treatment. Mayo Clin Proc 2017; 92:251-65.

2. Ighbariya A, Weiss R. insulin resistance, prediabetes, metabolic syndrome: what should every pediatrician know? I Clin Res Pediatr Endocrinol 2017; 9(Suppl 2):49-57.

3. Marginean CO, Melit LE, Ghiga DV, Marginean MO. Early Inflammatory Status Related to Pediatric Obesity. Front Pediatr 2019; 7:241.

4. Erdim I, Erdur O, Oghan F, Mete F, Celik M. Blood count values and ratios for predicting sleep apnea in obese children. Int I Pediatr Otorhinolaryngol 2017; 98:85-90.

5. Aydin M, Yilmaz A, Donma MM, et al. Neutrophil/lymphocyte ratio in obese adolescents. North Clin Istanb 2015; 2:87-91.

6. Abdel-Moneim A, Mahmoud B, Sultan EA, Mahmoud R. Relationship of leukocytes, platelet indices and adipocytokines in metabolic syndrome patients. Diabetes Metab Syndr 2019; 13:874-80

7. Milosevic D, Panin VL. relationship between hematological parameters and glycemic control in type 2 diabetes mellitus patients. I Med Biochem 2019; 38:164-71.

8. Wang YY, Lin SY, Liu PH, Cheung BM, Lai WA. Association between hematological parameters and metabolic syndrome components in a Chinese population. I Diabetes Complications 2004; 18:322-7.

9. Tao LX, Li X, Zhu HP, et al. Association of hematological parameters with metabolic syndrome in Beijing adult population: a longitudinal study. Endocrine 2014; 46:485-95.

10. Lee CT, Harris SB, Retnakaran R, et al. White blood cell subtypes, insulin resistance and beta-cell dysfunction in high-risk individuals--the PROMISE cohort. Clin Endocrinol (Oxf) 2014; 81:536-41.

11. Shiny A, Bibin YS, Shanthirani CS, et al. Association of neutrophil-lymphocyte ratio with glucose intolerance: an indicator of systemic inflammation in patients with type 2 diabetes. Diabetes Technol Ther 2014; 16:524-30.

12. Choi KM, Lee J, Kim YH, et al. Relation between insulin resistance and hematological parameters in elderly Koreans-Southwest Seoul (SWS) study. Diabetes Res Clin Pract 2003; 60:205-12.

13. Kim DJ, Noh JH, Lee BW, et al. The associations of total and differential white blood cell counts with obesity, hypertension, dyslipidemia and glucose intolerance in a Korean population. J Korean Med Sci 2008; 23:193-8. 
14. Neyzi O, Bundak R, Gokcay G, et al. reference values for weight, height, head circumference, and body mass index in Turkish children. J Clin Res Pediatr Endocrinol 2015; 7:280-93.

15. Demir K, Ozen S, Konakci E, Aydin M, Darendeliler F. A comprehensive online calculator for pediatric endocrinologists: CEDD Cozum/TPEDS metrics. I Clin Res Pediatr Endocrinol 2017; 9:182-4.

16. Tanner JM, Whitehouse RH. Clinical longitudinal standards for height, weight, height velocity, weight velocity, and stages of puberty. Arch Dis Child 1976; 51:170-9.

17. Kurtoglu S, Hatipoglu N, Mazicioglu M, Kendirici M, Keskin $M$, Kondolot $M$. Insulin resistance in obese children and adolescents: HOMA-IR cut-off levels in the prepubertal and pubertal periods. J Clin Res Pediatr Endocrinol 2010; 2:100-6.

18. Valerio G, Licenziati MR, lannuzzi A, et al. Insulin resistance and impaired glucose tolerance in obese children and adolescents from Southern Italy. Nutr Metab Cardiovasc Dis 2006; 16:27984.

19. Kuroda M, Sakaue H. Adipocyte death and chronic inflammation in obesity. I Med Invest 2017; 64:193-6.

20. Neels JG, Olefsky JM. Inflamed fat: what starts the fire? J Clin Invest 2006; 116:33-5.

21. Hotamisligil CS, Arner P, Caro JF, Atkinson RL, Spiegelman BM. Increased adipose tissue expression of tumor necrosis factoralpha in human obesity and insulin resistance. I Clin Invest 1995; 95:2409-15

22. Fernandez-Real JM, Vayreda M, Richart C, et al. Circulating interleukin 6 levels, blood pressure, and insulin sensitivity in apparently healthy men and women. / Clin Endocrinol Metab 2001; 86:1154-9.

23. Chmelar I, Chung KJ, Chavakis T. The role of innate immune cells in obese adipose tissue inflammation and development of insulin resistance. Thromb Haemost 2013; 109:399-406.

24. Talukdar S, Oh DY, Bandyopadhyay G, et al. Neutrophils mediate insulin resistance in mice fed a high-fat diet through secreted elastase. Nat Med 2012; 18:1407-12.

25. Hong X, Cui B, Wang M, Yang Z, Wang L, Xu Q. Systemic immune-inflammation index, based on platelet counts and neutrophil-lymphocyte ratio, is useful for predicting prognosis in small cell lung cancer. Tohoku J Exp Med 2015; 236:297-304.

26. Sun $X$, Liu $X$, Liu J, et al. Preoperative neutrophil-to-lymphocyte ratio plus platelet-to-lymphocyte ratio in predicting survival for patients with stage I-II gastric cancer. Chin J Cancer 2016; 35:57.

27. Bahadir A, Baltaci $D$, Turker $Y$, et al. Is the neutrophil-tolymphocyte ratio indicative of inflammatory state in patients with obesity and metabolic syndrome? Anatol / Cardiol 2015; 15:816-22.

28. Suarez-Cuenca JA, Ruiz-Hernandez AS, Mendoza-Castaneda AA, et al. Neutrophil-to-lymphocyte ratio and its relation with pro-inflammatory mediators, visceral adiposity and carotid intima-media thickness in population with obesity. Eur I Clin Invest. 2019; 49:e13085.

29. Vuong I, Qiu Y, La M, Clarke G, Swinkels DW, Cembrowski G. Reference intervals of complete blood count constituents are highly correlated to waist circumference: should obese patients have their own "normal values?". Am J Hematol 2014; 89:671-7.
30. Schafer Al. Thrombocytosis. N Engl ) Med 2004; 350:1211-9.

31. Maury E, Brichard SM, Pataky Z, Carpentier A, Golay A, BobbioniHarsch E. Effect of obesity on growth-related oncogene factoralpha, thrombopoietin, and tissue inhibitor metalloproteinase-1 serum levels. Obesity (Silver Spring) 2010; 18:1503-9.

32. Jones RL, Paradise $\mathrm{C}$, Peterson CM. Platelet survival in patients with diabetes mellitus. Diabetes 1981; 30:486-9.

33. Arslan N, Makay B. Mean platelet volume in obese adolescents with nonalcoholic fatty liver disease. I Pediatr Endocrinol Metab 2010; 23:807-13.

34. Aypak C, Turedi O, Bircan MA, Yuce A. Could mean platelet volume among complete blood count parameters be a surrogate marker of metabolic syndrome in prepubertal children? Platelets 2014; 25:393-8.

35. Erdal E, Inanir M. Platelet-to-lymphocyte ratio (PLR) and Plateletcrit (PCT) in young patients with morbid obesity. Rev Assoc Med Bras (1992) 2019; 65:1182-7.

36. Samocha-Bonet $D$, Justo $D$, Rogowski $O$, et al. Platelet counts and platelet activation markers in obese subjects. Mediators Inflamm 2008; 2008:834153.

37. Özsu E, Yazıcıoğlu B. Relationship between obesity and platelet indices in children. Cukurova Med I 2018; 43:30-5.

38. Hou J, Liu C, Yao P, et al. Association of adiposity indices with platelet distribution width and mean platelet volume in Chinese adults. PLoS One 2015;10:e0129677.

39. Park BJ, Shim JY, Lee HR, Jung DH, Lee JH, Lee YJ. The relationship of platelet count, mean platelet volume with metabolic syndrome according to the criteria of the American Association of Clinical Endocrinologists: a focus on gender differences. Platelets 2012; 23:45-50.

40. Kutlucan A, Bulur S, Kr S, et al. The relationship between mean platelet volume with metabolic syndrome in obese individuals. Blood Coagul Fibrinolysis 2012; 23:388-90.

41. Tavil Y, Sen N, Yazici HU, Hizal F, Abaci A, Cengel A. Mean platelet volume in patients with metabolic syndrome and its relationship with coronary artery disease. Thromb Res 2007; 120:245-50.

42. Furman-Niedziejko A, Rostoff $P$, Rychlak $R$, et al. Relationship between abdominal obesity, platelet blood count and mean platelet volume in patients with metabolic syndrome. Folia Med Cracov 2014; 54:55-64.

43. Budak YU, Polat M, Huysal K. The use of platelet indices, plateletcrit, mean platelet volume and platelet distribution width in emergency non-traumatic abdominal surgery: a systematic review. Biochem Med (Zagreb) 2016; 26:178-93.

44. Han S, Gan D, Wang G, et al. Associations of platelet indices with body fat mass and fat Distribution. Obesity (Silver Spring) 2018; 26:1637-43.

45. Furuncuoglu Y, Tulgar S, Dogan AN, Cakar S, Tulgar YK, Cakiroglu B. How obesity affects the neutrophil/lymphocyte and platelet/ lymphocyte ratio, systemic immune-inflammatory index and platelet indices: a retrospective study. Eur Rev Med Pharmacol Sci 2016; 20:1300-6.

46. Reddy UM, Khandekar A, Acharya S, Shukla S, Acharya N Study of platelet indices in patients with metabolic syndrome. Medical and Surgical Update Society 2019; 05:10-4. 
47. Qi YL, Zhang Y, Zhao LD, Wang AX, Wang ZB, Gao QL. Platelet to lymphocyte ratio in peripheral blood and body mass index: novel independent prognostic factors in patients with melanoma]. Zhonghua Yi Xue Za Zhi 2017; 97:3704-10.

48. Yu JY, Choi WJ, Lee HS, Lee JW. Relationship between inflammatory markers and visceral obesity in obese and overweight Korean adults: An observational study. Medicine (Baltimore) 2019; 98:e14740.
49. Helou MA, Sisler I, Ning Y, Liu H. Is obesity alone associated with Increased blood cell counts in otherwise healthy children? Blood 2011; 118:3135. 